\title{
Thermopile Sensor Array with Improved Spatial Resolution, Sensitivity and Image Quality
}

\author{
B. Forg, F. Herrmann, J. Schieferdecker, W. Leneke, M. Schulze, M. Simon, K. Storck (Heimann Sensor \\ $\mathrm{GmbH}$ ), F. Völklein (University of Applied Sciences, Wiesbaden) \\ Heimann Sensor GmbH \\ Grenzstrasse 22 \\ 01109 Dresden \\ Hochschule RheinMain \\ University of Applied Sciences \\ Kurt-Schumacher-Ring 18 \\ 65197 Wiesbaden
}

\begin{abstract}
:
Heimann Sensor announces the new HTPA64x62 infrared image sensor. The new sensor array is pin-topin compatible to the existing HTPA32x31. The thermopile-based HTPA series provides sensor elements with very low time constants and high sensitivity, signal processing with a high frame rate comprising low noise amplifiers and serial peripheral interface. Some types have the option to provide digital image data by an integrated analogue to digital converter.

On the basis of our several years experience in design and development of thermopile sensors we improved the sensitivity of existing image sensor HTPA32x31 by a factor of $8 \ldots 10$ using advanced pixel design combined with vacuum packaging. Even if vacuum has been replaced by an inert gas filling the sensitivity is increased by a factor of $4 \ldots 6$ compared to our existing designs. Inert gas filling has the advantage of a reasonable sensitivity combined with lower time constant of $7 \mathrm{~ms}$ only. This paper deals with the simulation and test results of our new thermopile array technology.
\end{abstract}

\section{Introduction:}

Thermopile sensor arrays are a cost competitive solution in comparison to other technologies, wherever a thermal image of the environment is needed and where very high spatial resolutions are not necessary, such as person detection, surveillance of critical surface temperature, hotspot detection, energy management and security cameras. Other applications can be found in industrial process control, air condition control, fever detection of travelling passengers and fire detection / protection.

The benefits of this technology are the low costs, the very low power consumption, the short time constant of the elements, as well as the high sensitivity of the system.

Heimann Sensor announced the first fully monolithic $8 \times 8$ and 16x16 thermopile arrays in 2008 [1] and improved this technology to $32 \times 31$ sensitive elements a year later. As result of our consistent further development the new sensor array of 64x62 sensor elements is now announced in year 2011.

\section{Theory}

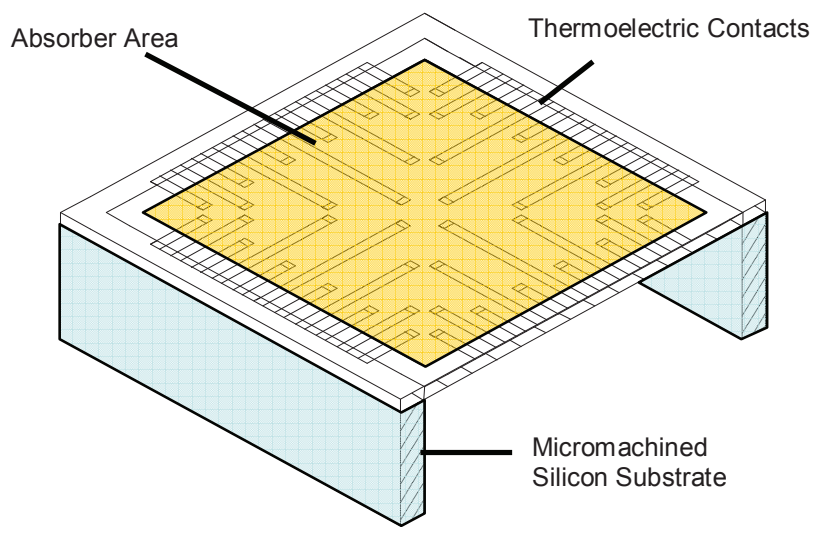

Fig.1: Standard thermopile pixel
A thermopile is a series of thermocouples, structured on a thin membrane. All designs have the common attribute, that the "cold" contacts are structured on a heat sink, which is usually a thick silicon bar and the "hot" contacts are on a thin membrane. If the absorber on the membrane is exposed to a hot object, the radiation flow between membrane and object will heat the membrane up. This will cause the thermopile to create a voltage due to the Seebeck effect. For conservative pixel designs, the thermal conductivity of the membrane is the most dominating effect of heat transfer from hot (absorber area of membrane) to cold contacts (substrate area) of a pixel. 
Regarding Fourier's law the heat flow rate can be described as $\frac{\Delta Q}{\Delta t}=-k \cdot A \frac{\Delta T}{\Delta x}$ where $A$ is the cross sectional surface area, $\Delta T$ is the temperature difference, $\Delta x$ is the distance $k$ is the materials conductivity. Therefore it is obvious that the heat flow rate can be decreased by a small cross sectional surface area or a long distance. Minor heat flows result in a larger temperature gradient across the membrane, which causes higher voltages and improves the signal to noise ratios. If the heat transfer through the membrane gets low, the thermal conductivity of the surrounding gas gets major heat transfer mechanism. Consequently, the sensitivity can be further increased by using a gas with a lower thermal conductivity.

\section{Results}
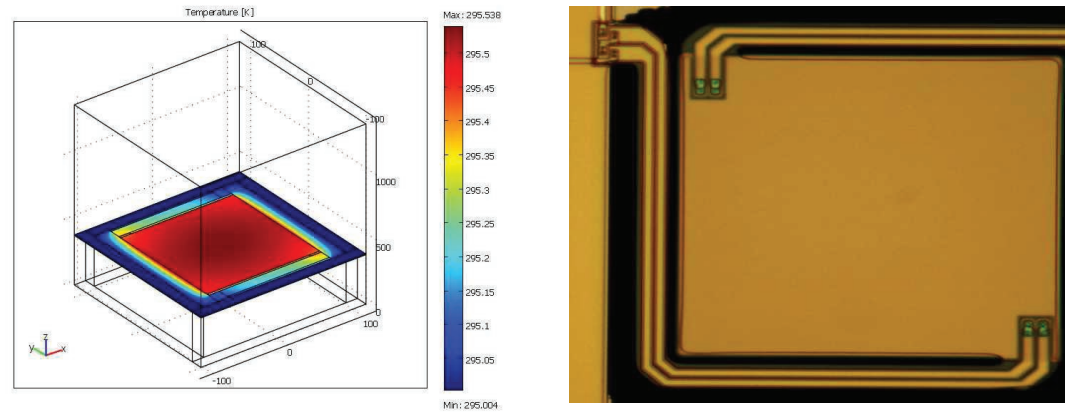

The new technology for higher sensitivity pixels is shown in Fig 2a (thermal simulation) and Fig. 2b (layout photo). Only very few thermocouples are designed as long beams on a reticulated membrane.

Fig. 2: a) Thermal simulation b) photo of prototyped membrane

As already mentioned if the thermal conductivity of surrounding gas dominates the heat transfer, the sensitivity can be significantly increased using gases of low conductivity like $\mathrm{Xe}$ or $\mathrm{Ar}$ or even vacuum environment in the sensor housing. Simulation shows a signal increase by about factor $3 \ldots 4$ for Xenon filling or up to factor 20 for vacuum. Sensitivity rising is accompanied by increased response time of $8 \mathrm{~ms}$ for Xenon or about $50 \mathrm{~ms}$ for vacuum. Measured sensitivity values in vacuum reached up too $1500 \mathrm{VM}$.

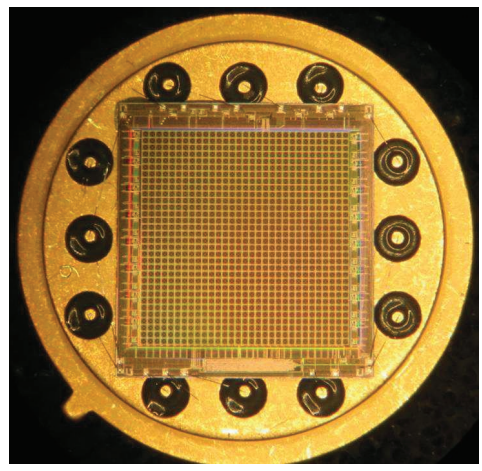

The $8.5 \times 9 \mathrm{~mm}^{2}$ monolithic array chip with signal conditioning is mounted on a TO-8 header (Fig. 3). In general we used our effective HTPA32x31 design and decreased the pitch of the sensitive elements to $110 \mu \mathrm{m}$ to create the HTPA64x62. Since the distance between the hot and cold contacts is decreased in this design, this also affects the output signal of a pixel. Therefore we decided to use a vacuum sealing for the HTPA64x62 as standard and an inert gas filling for the HTPA32×31. Due to the small temperature gradient of the sensitivity from the ambient temperature, there is no temperature stabilization of the chip necessary. The thermoelectric materials are $n$ - and $\mathrm{p}$-doped poly silicon, which require a modified CMOS-Process. Power consumption of the array itself is about $35-50 \mathrm{~mW}$ only, depending on type and frame rate.

Fig. 3: HTPA32×31 mounted on a TO8 base plate

For optimization purposes we simulated different pixel designs with miscellaneous beam lengths and shapes. Furthermore we considered the influence of the different gas fillings. In Fig. 4 one of these simulation results can be seen: The y-axis shows the temperature of the membrane in dependency of the cross section in the $x$ coordinate. Parametric the different cross sections in the y coordinate of the pixel can be seen.

The thermal noise can be calculated by $v_{N}=\sqrt{4 k T R \Delta f}$, where $k$ is the Boltzmann's constant, $T$ is the absolute temperature, $\Delta f$ is the bandwidth and $\mathrm{R}$ is the resistance.

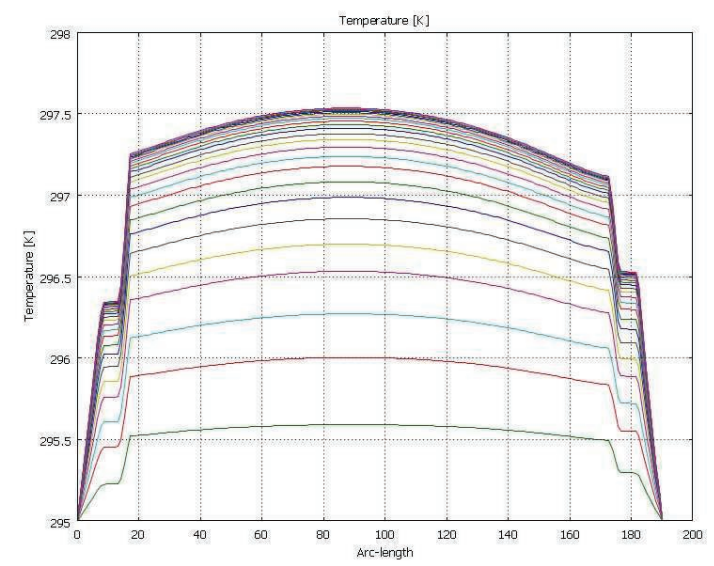

Fig. 4: Temperature of the membrane in dependency of the $x$ coordinate 
As a conclusion of our results we optimized the number of thermocouples per pixel. It can be easily seen, that with an increasing number of thermocouples and therefore with rising pixel impedance, the thermal noise increases, too. Since the heat conductivity enhances with the number of thermocouples (because of the growing cross section of poly silicon) there is an optimum for the sensitivity which can be reached.

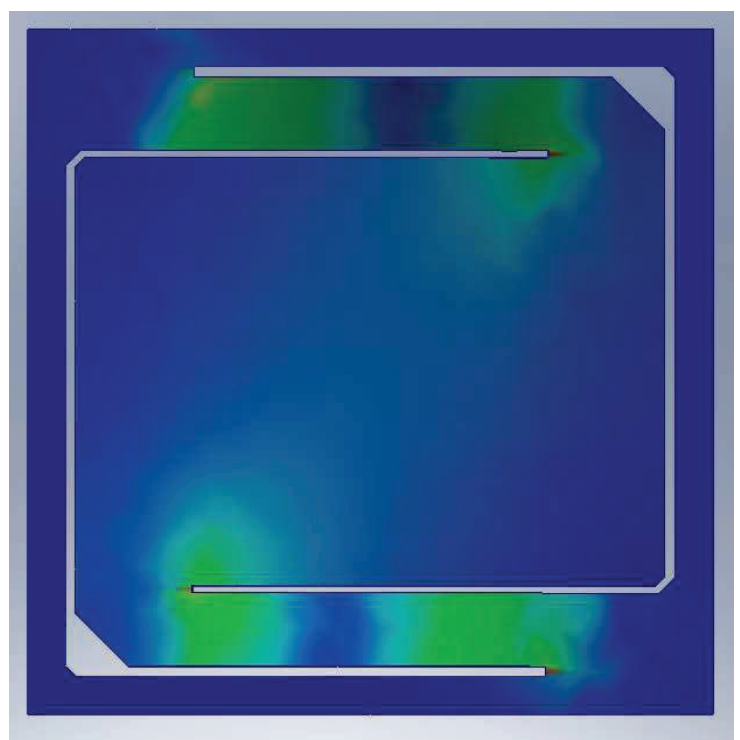

Furthermore we have done simulations to determine the critical stress of the pixel designs. This was necessary for designing a pixel which can withstand great accelerations, which is the most important parameter to avoid broken membranes in the field, i.e. in harsh environments. In Fig. 5 a result of these simulations is shown as false colour diagram of the resulting vonMises stress in the membrane.

These new arrays complete our HTPA series. Both the HTPA32 $\times 31 \mathrm{Hi}$ with improved sensitivity, as well as the HTPA64x62 could be demonstrated and measured. Furthermore, we designed new optics for arrays. Consequently, four HTPA types with different pixel numbers can be combined with more than 10 single lenses or optical systems to adapt the array to the required field of view in the application - e.g. a two lens optics with aspherical surfaces, the L10/0.8 (focal length

Fig. 5: von-Mises stress as false colour diagram $=10 \mathrm{~mm}$; numerical aperture $=0.8$ ).
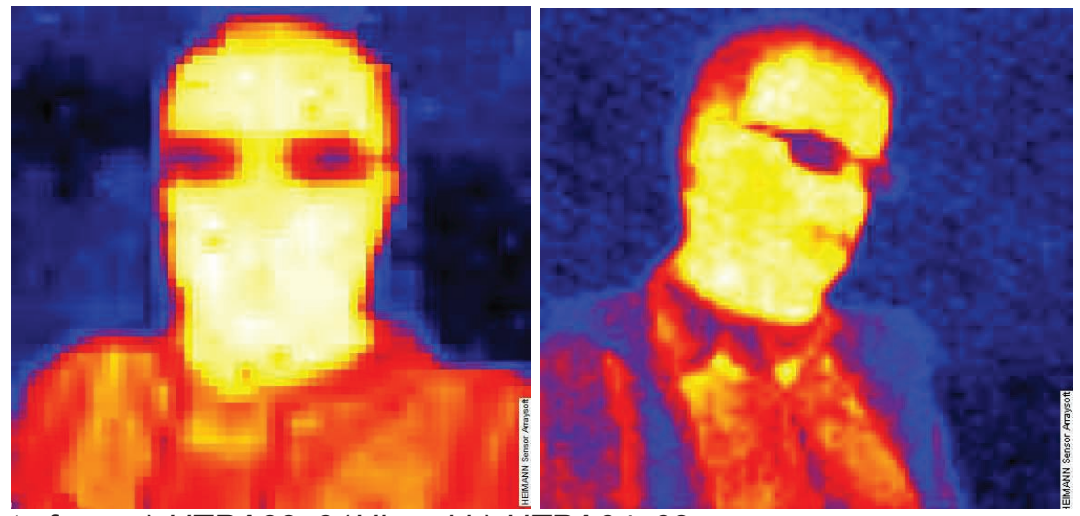

Fig. 6: Screenshots from a) HTPA32x31Hi and b) HTPA64x62

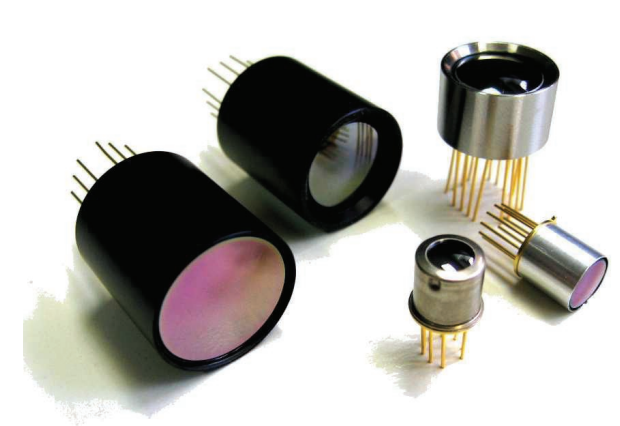

Fig. 7: HTPA series types with different optics

The HTPA8x8 is now available in the small TO39 package with only approx. $8 \mathrm{~mm}$ diameter housing. This miniaturized array provides a perfect solution for low cost applications, since the diameter of the optics is a cost driving factor. Some examples of arrays with different packages and optics can be seen in Fig. 7 .

We also expanded the HTPA module portfolio. Our HTPA modules provide an easy way to get a calibrated temperature stream from the sensor. Heimann Sensor developed modules with SPI, UDP and UART interface. A CAN interface will be available, soon. Furthermore, we developed a SDK (Software Development Kit) for the SPI module to have an easy and comfortable application.

[1] B. Forg, W. Leneke, J. Schieferdecker, M. Schulze, M. Simon, K. Storck:

"Thermopile Sensor Arrays with Internal Amplifiers and Digital Out"; Sensor \& Test 2008, Nürnberg, Proceedings, p. 249-252. 